Ann. Zootech., I967, 16 (4), 323-334.

\title{
HYPOTHÈSE GÉNÉTIQUE UNIQUE POUR EXPLIQUER LA PRÉSENCE D'INTERSEXUÉS, DE MÂLES EN EXCÈS ET DE MÂLES STÉRILES EN RACE CAPRINE SAANEN.
}

\author{
G. RICORDEAl, J.-J. IAUVERGNE \\ avec la collaboration de J. Bouiluon, Directeur de la Station de Testage \\ caprin de Moissac (Lozère) et de $\Lambda$. Lajous, technicien I. N. R. A. \\ Station centrale de Génétique animale, \\ Centre national de Recherches zootechniques, 78 -Jouy-en-Josas \\ Institut national de la Recherche agronomique
}

\section{SOMMAIRE}

Nous avons tout d'abord vérifié à nouveau qu'en race Saanen l'absence de cornes était due à un gène autosomal dominant et qu'il existait une différence de fécondité en faveur des chèvres mottes P'p par rapport aux homozygotes $p p$. Nous avons également décelé chez les mâles une influence nette mais non significative du génotype sur la taille des portées engendrées, les boucs PP semblant présenter un avantage sur les boucs $P$ p

Pour expliquer à la fois l'apparition de máles stériles et d'intersexués et le décalage du rapport des sexes dans les croisements entre géniteurs sans cornes, nous proposons une hypothèse unique selon larjuelle les femelles PP' sont masculinisées d'une manière plus ou moins complète pour donner soit des intersexués, soit des animaux stériles d'apparence mâle classés parmi les boucs. Ainsi le gène autosomal motte aurait, outre son effet dominant à pénétrance complète dans les deux sexes pour l'absence de cornes, un effet récessif de masculinisation limité au sexe femelle. Des données recueillies pendant trois amnées dans la zone du Syndicat caprin de Sainte-Croix-Vallée-Française et des résultats de testage de la stérilité des mâles permettent une vérification statistique de cette hypothèse. Un examen caryologique reste nécessaire pour conclure définitivement en précisant le sexe génétique des mâle's stériless.

\section{IN'TRODUC'TION}

La question de l'intersexualité associée à l'absence de cornes domine la génétique de l'espèce caprine đans les pays occidentaux depuis plus d'un quart de siècle. Les recherches nombreuses qui ont été effectuées se rapportent toutes au caractère 
motte (1) originaire des Alpes, semble-t-il, qui a diffusé un peu partout dans le monde avec la vogue des chèvres suisses. Après avoir étudié indépendamment l'hérédité du cornage, puis celle de l'intersexualité, les auteurs ont admis qu'il existait une liaison génétique entre les deux caractères. Une première hypothèse simple ne s'étant pas révélée suffisante, il a fallu chercher d'autres explications aux écarts systématiques dans le rapport des sexes. Voyons successivement plus en détail ces différentes étapes.

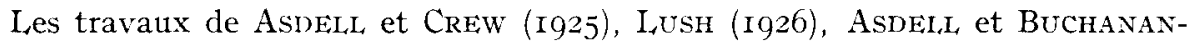
Smiтh (I928), montrent tout d'abord que le caractère d'absence de cornes est commandé par un gène dominant autosomal.

L'intersexunlité, présente dans les races d'origine alpine et décrite depuis longtemps, reçoit un clébut d'interprétation génétique avec FUNKQUIST (I932), puis avec EATON et Srmmons (I939). Ces derniers auteurs concluent provisoirement que 1'anomalie est due à un gène autosomal récessif. Cependant, on avait déjà observé que l'intersexualité existait dans les souches où l'on sélectionnait contre la présence de cornes et, mieux encore, qu'elle se manifestait uniquement sur des animaux mottes, à quelques rares exceptions près. Iin Grande-Bretagne et aux États-Unis, à partir de I94I, l'idée d'une liaison entre le déterminisme génétique des deux phénomènes s'est faite jour peu à peu. Fn I944, ASDEI, , présente l'hypothèse selon laquelle l'intersexualité est due à un gène récessif situé très près du gène dominant pour le caractère motte et dont l'action est limitée au sexe femelle. Cette dernière clause semble expliquer le déséquilibre du rapport des sexes en faveur des mâles. FATON (I945) pense avoir vérifié la nouvelle hypothèse à partir des données provenant des chèvres Saanen et Toggenbourg de Beltsville.

Par la suite, comme l'avait suggéré Lush à AsDei, , (Asilel, I 946), l'hypothèse de deux gènes très proches sur le même chromosome a été abandonnée en faveur de l'hypothèse d'un gène unique à effet pléiotropique, dominant pour l'absence de cornes dans les deux sexes, récessif pour l'intersexualité dans le sexe femelle uniquement. Des preuves cytologiques ont été fournies ultérieurement montrant que le sexe génétique des animaux intersexués était bien femelle (NFS, ANIERSFN et SI,AGSVoLD, 1963; Basrur et Coubrough, I964; de Grouchy, Lauvergne et Ricordeau, I965).

Cette dernière hypothèse n'est cependant pas entièrement satisfaisante comme le montre BUECrI (1957) dans les races mottes suisses, puisque, même en ajoutant les intersexués aux femelles, le taux de masculinité reste supérieur à la normale. Selon cet auteur, le déséquilibre persistant du rapport des sexes en faveur des mâles serait dû̀ à une plus grande mortalité des embryons femelles nottes au cours des premiers stades de la gestation.

I,es chercheurs israéliens, à partir de LAOR et al. (I962) vont apporter une contribution précieuse à l'étude de ce problème en associant aux cas des intersexués celui des mâles stériles, phénomène connu depuis longtemps, mais qui avait été aborclé séparément et presque uniquement par des auteurs allemands (cf. revue de SchöNHERR, I956 et LöHLE et BARruss ig5r, par exemple). SOr.,ER et al. (I963) pensent que, si les femelles mottes homozygotes sont transformées en intersexués, la stérilité peut également affecter certains mâles homozygotes mottes avec, dans ce cas, une pénétrance incomplète. Conme cette théorie n'explique pas toutes les ano-

(1) Sans cornes. 
malies observées et que la proportion de "femelles génétiques " (nombre de femelles et d'intersexués pour roo mâles) qui varie avec le type d'accouplement, semble augmenter avec l'importance de la portée, SOLLER et ANGEr, (1964) invoquent un "effet récessif du gène motte sur le rapport des sexes ». D'après ces deux auteurs, les variations observées dans la proportion de "femelles génétiques " peuvent être, en outre, attribuées soit à une disparition précoce d'embryons femelles mottes (hypothèse déjà formulée par BUECHI, I957), soit à une masculinisation totale en apparence de certaines femelles génétiques homozygotes mottes.

L'hypothèse d'une élimination précoce des embryons femelles mottes n'est guère soutenable : en effet, les chèvres mottes hétérozygotes se révèlent, en fin de compte, plus fécondes que les chèvres cornues de même origine (SOLI_ER et KEMPE$\mathrm{NICH}$, I 644 ) bien qu'elles donnent, en moyenne, plus de descendants sans cornes. Quant à l'hypothèse d'une action récessive du gène sur le rapport des sexes, nous pensons qu'elle s'efface devant l'hypothèse unique selon laquelle toutes les femelles $\mathrm{PP}$ sont masculinisées soit en intersexués, soit en mâles stériles. Cette hypothèse unique repose sur le fait que, l'expressivité du phénomène d'inversion du sexe étant extrêmement variable (EATON, I943; KONDO, I949), on peut très bien concevoir que certaines femelles, masculinisées très tôt dans la vie embryonnaire, parviennent à un stade de ressemblance parfaite avec les mâles, tout en demeurant, bien entendu, incapables de produire des gamètes fertiles.

Pour vérifier cette hypothèse nous avons utilisé les données enregistrées en Lozère pendant trois campagnes. Ces données nous permettent, au passage, de vérifier le déterminisme héréditaire du cornage et de préciser l'influence des différents génotypes sur le taux de fécondité.

\section{I. - MA'TÉRIEL E'T MÉTHODES}

Les données ont été recueillies dans la zone du Syndicat caprin de Sainte-Croix-Vallée-Française (Lozère). La population caprine y est composée d'animaux issus d'accouplements de chèvres de pays avec des boucs Saanen originaires de Sarre, dont l'introduction remonte à 1958 .

Depuis cette date, un contrôle des performances a été mis en place, qui permet d'enregistrer systématiquement les ascendances, les mises bas et les performances laitières. Ces trois dernières années on a, en plus, noté le cornage et l'intersexualité. Les données utilisables concernent I $73^{2}$ mises bas et 3007 produits.

Quant au cornage, on a éliminé les renseignements douteux par suite d'enregistrements incomplets et de mortalité natale ou péri-natale ; on s'est attaché, en outre, à le vérifier à plusieurs stades. En effet, les cornes apparaissent plus ou moins tardivement après la naissance et les enregistrements effectués dès la mise bas risquent de sous-estimer la fréquence des animaux cornus. Nous avons pu constater notamment que la présence d'un épi sur chaque bourgeon corné indique presque sûrement la présence ultérieure de cornes (RICORDEAU et al, ig68).

De 1965 à 1967,73 boucs ont été utilisés : 1o comus et 63 mottes. Ayant examiné suffisamment de descendants de boucs mottes, on a pu déterminer individuellement leur génotype : so, n'ayant donné aucun descendant cornu, doivent être considérés comme homozygotes sans cornes (PP), les 53 autres étant hétérozygotes $\mathrm{P} p$ (parmi ceux-ci, l'un portait des cornillons).

Les auteurs précéclents ont appelé $\mathrm{P}$ (de l'anglais polled) le gène pour l'absence de cornes. Cette appellation est impropre, car $\mathbf{P}$ désigne déjà chez les rongeurs le locus " pink eyed". La terminologie des loci de la chèvre étant inexistante, nous conserverons cependant cette nomenclature, en désignant par [P] le phénotype sans cornes. Pour les types d'accouplements, nous utiliserons la classification adoptée par LAOR et al. (1962).

Quant à la fécondité, on a tenu compte de toutes les naissances. I e taux de fécondité est exprimé par le nombre de produits obtenus pour roo mères qui ont mis bas. 


\section{II. - - VÉRIFICA'TION DE L'HYPO'THËSE DE DOMINANCE AUTOSOMALE DE I'ABSENCE, DE CORNES}

Les résultats concernant la vérification de cette hypothèse sont contenus dans le tableau I.

On suppose tout d'abord que tous les boucs cornus sont homozygotes $p p$ : accouplés à des femelles cornues, également $p p$ (type d'accouplement $n^{\circ} 6$ ), ils ne doivent donner que des descendants cornus, c'est en effet ce que 1'on observe.

Les boucs mottes qui n'ont jamais donné de produits cornus sont classés comme homozygotes $\mathrm{PP}$, on les retrouve dans les accouplements I et 4.

Les fréquences des descendants cornus dans les types d'accouplements 2 et 3 ne sont pas significativement différentes respectivement de 25 et $50 \mathrm{p}$. I 00 . La fréquence du gène $\mathrm{P}$ étant très voisine de 0,5 , cela signifie que presque toutes, sinon toutes les chèvres sans cornes fertiles sont hétérozygotes.

Pour les accouplements 3 et 5 (l'un des parents est cornu, et l'autre motte hétérozygote), la proportion des descendants cornus est presque égale au pourcentage théorique espéré $(50,4 \mathrm{p}$. IOo), mais le type 3 (père cornu $\times$ mère motte) donne plus de produits cornus que le type 5 (père motte $\times$ mère cornue) ; les écarts ne sont cependant pas significatifs.

Ainsi, le déterminisme monofactoriel autosomal dominant du facteur pour l'absence de cornes peut-il être considéré comme vérifié.

\section{III. - ACTION DU GÉNOTYPE DE LA MÈRE E'T DU GÉNOTYPE DU PÈRE SUR LA FÉCONDITÉ}

Comme, probablement, toutes les femelles mottes sont hétérozygotes ( $\mathrm{P} p)$, il est facile de comparer la fécondité des femelles de ce génotype (toutes celles qui sont classées dans les accouplements de type I, 2 et 3) à celles de génotype homozygote récessif $p p$. Les résultats moyens résumés dans le tableau 2 montrent que les femelles mottes ont un taux de fécondité supérieur à celui des femelles cornues, ce qui confirme les observations de SOI,LER et KEMPENich (I964).

Il semble aussi exister une influence du génotype du mâle sur la fécondité $\left({ }^{1}\right)$. En effet, si l'on compare les accouplements de type I et 3 , et ceux du type 4 et 6 , dans lesquels les femelles sont de même génotype, on constate que le taux de fécondité des mâles PP est supérieur à celui des mâles $p p$, alors que les taux de fécondité des accouplements réciproques 3 et 5 sont tout à fait comparables (tabl. 2). Il s'agit là, en fait, de résultats bruts qui ne tiennent pas compte des différences annuelles, de l'âge des chèvres et de l'influence du "niveau d'élevage ". Toutefois, même si les écarts observés

(1) Il est intéressant de noter à ce sujet les différences observées dans les carartéristiques des éjaculats de boucs appartenant aux 3 races françaises (CORTEEL, I 966 ). 


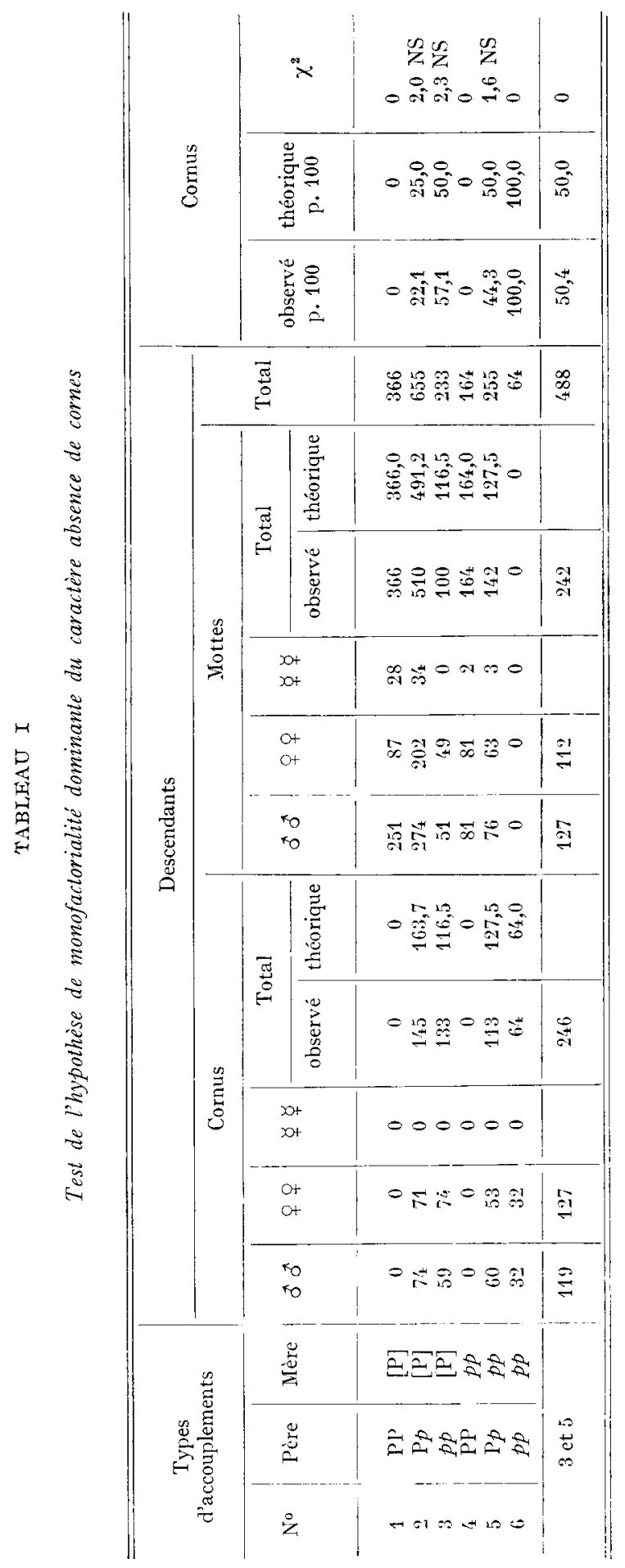


TABLEAU 2

Taux de fécondité comparés des femelles $P \mathrm{p}$ et $\mathrm{pp}$ et des mâles $P P$ et $\mathrm{pp}$

\begin{tabular}{|c|c|c|c|c|c|}
\hline \multicolumn{3}{|c|}{ Types d'accouplements } & \multicolumn{2}{|c|}{ Nombre de } & \multirow{2}{*}{$\begin{array}{l}\text { Taux de } \\
\text { fécondité } \\
\text { p. } 100\end{array}$} \\
\hline No & Père & Mère & mises-bas & chevreaux nés & \\
\hline 1 & PP & [P] & 219 & 404 & 184,5 \\
\hline 2 & $\mathrm{Pp}$ & [P] & 789 & 1366 & 173,1 \\
\hline 3 & $p p$ & [P] & 234 & 405 & 173,1 \\
\hline 4 & $\mathrm{PP}$ & $p p$ & 100 & 178 & 178,0 \\
\hline 5 & $\mathrm{P} p$ & $p p$ & 299 & 518 & 173,2 \\
\hline 6 & $p p$ & $p p$ & 91 & 136 & 149,5 \\
\hline Total & & & 1732 & 3007 & 173,6 \\
\hline
\end{tabular}

Différences pondérées du taux de fécondité entre femelles mottes et comues :

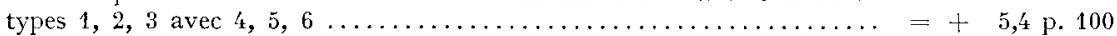

- entre mâles PP et $p p\left\{\begin{array}{l}\text { type } 1 \text { avec } 3 \ldots \ldots \ldots \ldots \ldots \ldots \ldots \ldots \ldots \ldots \ldots \ldots \ldots \ldots \ldots \ldots \ldots \ldots \ldots \ldots \ldots \ldots \ldots \ldots \ldots \ldots \\ \text { type } 4 \text { avec } 6\end{array}\right.$

ne sont pas significatifs, ils suggèrent d'entreprendre une étude plus précise de la fertilité des mâles, en saillie naturelle et surtout dans les conditions de l'insémination artificielle, comme cela a été fait par PeтrTJEAN et Cochez (Ig66) sur des coqs de race Wyandotte classés suivant le génotype conditionnant la forme de la crête.

\section{IV. - EXAMEN DE L'HYPOTHÈSE SELON LAQUELLE TOUTES LES FEMELI,ES HOMOZYGOTES POUR L'ABSENCE DE CORNES SONT TRANSFORMÉES EN INTERSEXUÉS OU EN MALES STÉRILES}

Nous voyons, tout d'abord, que tous les intersexués sont mottes. Ils n'apparaissent, à quelques exceptions près, que dans les croisements où nous les attendons : ceux où les deux parents sont de phénotypes sans cornes (tabl. 3). Les exceptions observées dans les croisements 4 et 5 (0,5 p. roo du total des naissances), peuvent s'expliquer par des erreurs d'ascendance ou par des anomalies chromosomiques (PADEH et al., I965).

La vérification de notre hypothèse repose sur les faits suivants.

I. Statistiquement, toutes les femelles fertiles mottes peuvent être considérées comme hétérozygotes, ce qui permet de déduire que les femelles PP sont impropres à la reproduction (cf. résultat énoncé plus haut à partir du tabl. I).

2. Dans les accouplements de type I et 2 , la proportion de femelles mottes correspond uniquement à celle des femelles $\mathrm{P} p$ attendues (tab1. 3) .

3. Si toutes les femelles PP sont stériles, les mâles PP, par contre, semblent se reproduire normalement. En effet, sur II fils du bouc 59002 (cf. tabl. 4) nés de 


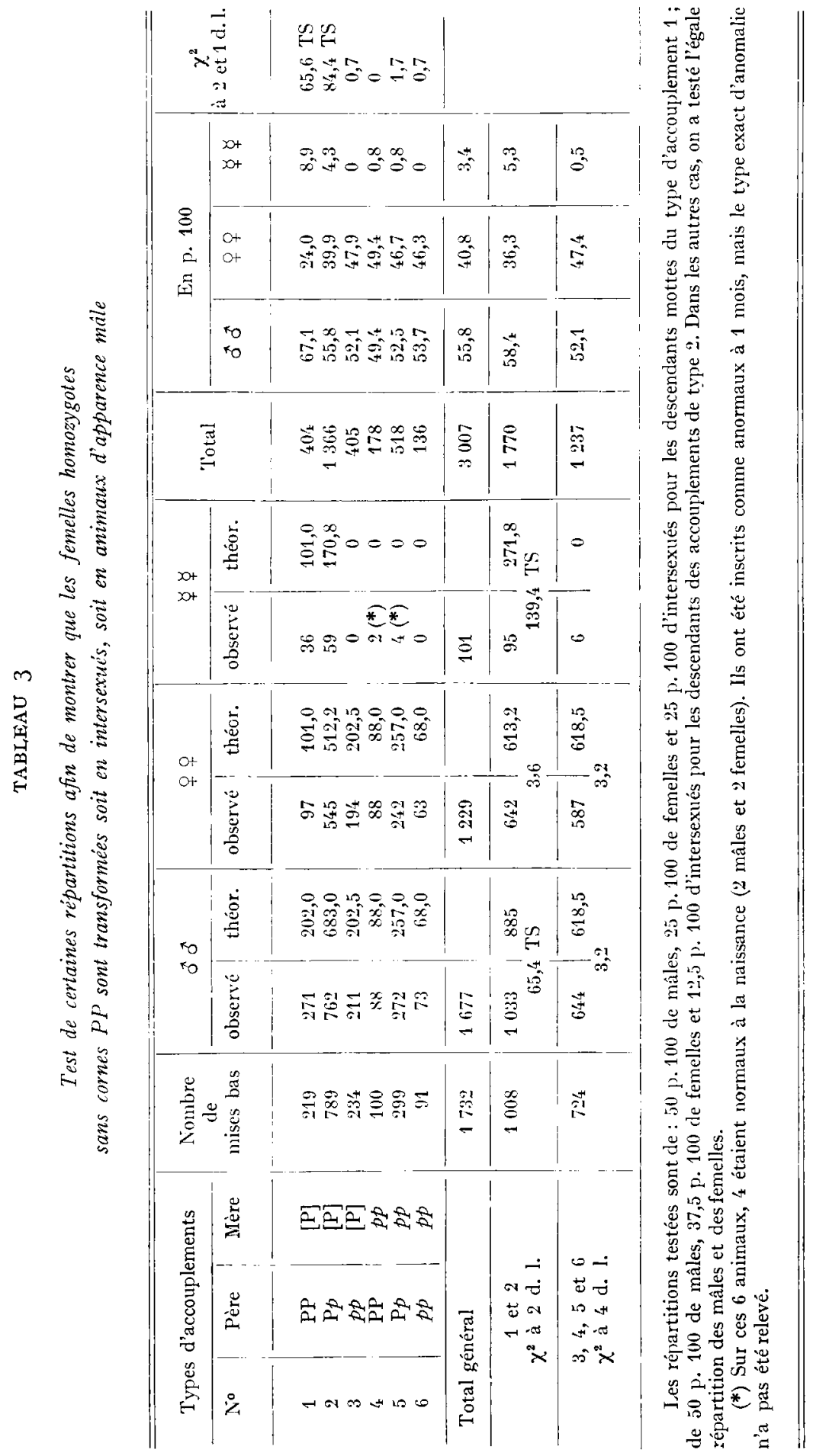


mères mottes et ayant fécondé normalement pendant au moins une saison, 4 ont été testés homozygotes PP avec, respectivement 19, 30, 52 et 66 produits. Compte tenu de la dimension de l'échantillon, la proportion observée est peu différente de la proportion théorique (50 p. IOO).

4. On observe les intersexués dans les deux seuls types d'accouplements susceptibles de fournir des homozygotes PP : les accouplements I et 2 . Ils sont inférieurs en nombre aux femelles PP attendues (tabl. 3).

5. Dans les accouplements I et 2, la proportion de mâles est significativement supérieure à $50 \mathrm{p}$. Ioo et on observe un certain nombre de mâles stériles ou cryptorchides. La fréquence de ces derniers a pu être évaluée parmi 23 descendants des accouplements de type I (tabl. $4 a$ et $b$ ) ; elle est de $26, \mathrm{I}$ p. Ioo, alors que la proportion de mâles en excès est de $2 I, 9$ p. IOO. La proportion des mâles stériles ou cryptorchides correspond donc à celle des mâles en excès. Si on l'ajoute à celle des intersexués, on retrouve exactement le pourcentage de femelles PP attendues.

\section{TABLEAU 4}

Examen de la descendance de 3 boucs I'P

a) Rapport des sexes sur 3 années consécutives

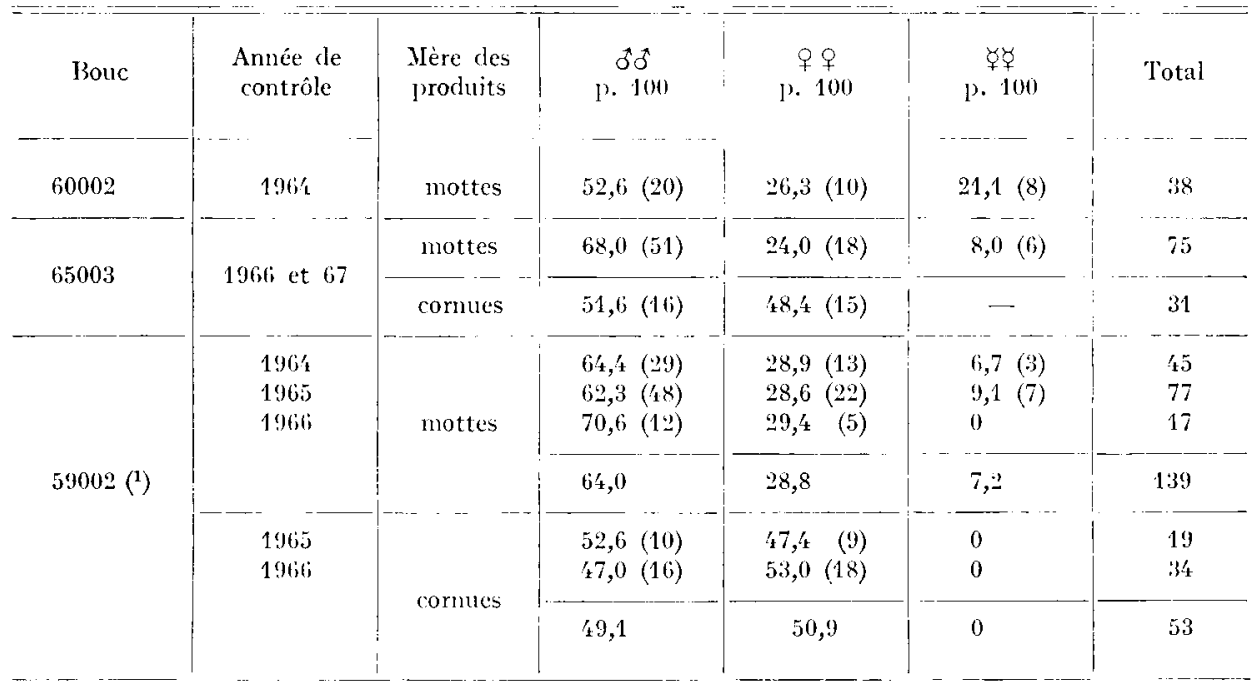

b) Examen des produits nés en $196 \pm d u$ bouc 59002 ( $\left.\mathrm{Pl}^{\prime}\right): 45$ produits, issus des 25 chèvres mottes, élevés pendant un an.

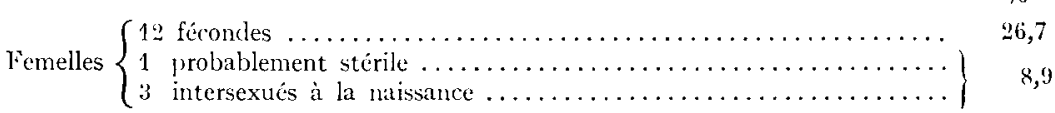

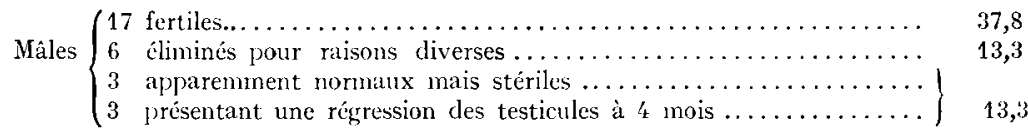

(1) Ce bouc est égalenent homozygote blanc. 
En reprenant maintenant les données d'auteurs qui ont classé les accouplements selon le génotype des parents (EATON, I945; HAugen, I960; SOLlER et ANGEr., I 964 ; tabl. 5) on observe une constance dans la fréquence des femelles mottes issues des croisements de type I et 2, alors qu'au contraire, la fréquence des intersexués est fort variable.

\section{TABI,EAU 5}

Résultats antérieurs concernant les accouplements de type 1 it 2

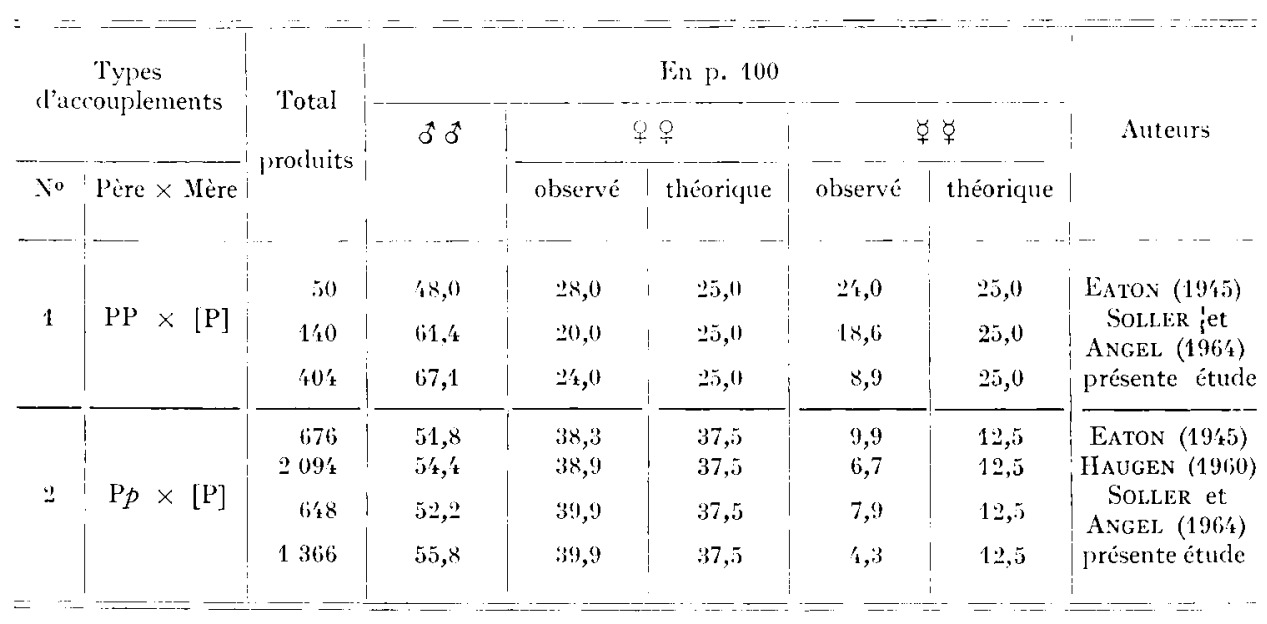

Le taux constant auquel se fixe la proportion des femelles mottes indique que très peu de femelles PP échappent à un début de masculinisation et sont confondues avec les hétérozygotes. On a pu, cependant, observer quelques rares cas de femelles apparemment normales à la naissance qui se masculinisent vers l'âge de 4 à 5 mois, avec renflement de la partie inférieure des lèvres de la vulve (bulbe rejeté en arrière) et apparition d'une barbiche. In outre, toutes les chevrettes soumises à la reproduction ne sont pas fécondes.

Les écarts dans les fréquences d'intersexués observés par les différents auteurs peuvent être dus à l'expressivité changeante de la masculinisation et à la difficulté de déceler certaines anomalies à la naissance. La proportion des intersexués sera d'autant plus élevée que les auteurs auront eu la possibilité de suivre tous les animaux jusqu'à un âge plus avancé. Ainsi, en élevant des jeunes mâles mottes issus de parents mottes, on observe deux catégories d'animaux : les premiers ont des testicules qui régressent vers 4-5 mois, les seconds sont apparemment normaux mais restent stériles. Cette observation, déjà signalée dans le tableau $4 b$, a été vérifiée en Ig66 et Ig67 dans la descendance de 2 I pères différents (sur 42 mâles mottes on a trouvé 5 cas de régression des testicules et 4 cas de stérilité). Lorsque les enregistrements sont faits en ferme, les statistiques concernant les anomalies sont forcément établies par défaut, car presque tous les mâles sont vendus à la boucherie à 2 ou 3 semaines. Les enregistrements sont également incomplets lorsqu'on se contente de contrôler la fertilité des boucs apparemment normaux, puisque les cryptorchides ont été éliminés au préalable. Pour détecter tous les mâles anormaux, le moyen le plus sûr serait donc 
d'établir systématiquement le caryotype des produits mâles issus des accouplements I et 2 , ce qui apporterait en même temps une vérification défnitive de notre hypothèse.

A l'appui de celle-ci nous avons uniquement considéré les accouplements I et 2, seuls susceptibles de produire des femelles PP. On a vérifié, en outre, que les accouplements 3 à 6 (tabl. 3) donnent un rapport des sexes non significativement différent de I : I.

\section{CONCLUSION}

Sous réserve de certaines vérifications, notre hypothèse explique aussi bien nos données d'observation, que celles d'autres auteurs. Les Israéliens qui ont récemment beaucoup fait progresser cette recherche n'ont cependant pas abouti à une explication simple. Ils ont eu conscience des liaisons existant entre l'apparition de mâles stériles et d'intersexués, mais ils ont considéré le décalage du rapport des sexes comme une anomalie particulière, alors que ce décalage s'explique simplement par l'expressivité variable du phénomène de masculinisation des femelles PP.

Du point de vue pratique, l'existence d'un tel gène d'absence de cornes associé à la masculinisation de certaines femelles dans une population caprine réduit considérablement la proportion des reproductrices possibles. En effet, les types I et 2 qui, en Lozère, représentent plus de la moitié des accouplements (6I p. roo en I964-65, 54 p. Ioo en I 666 ) entraînent finalement un déficit de I7 à I 8 femelles pour Ioo mises bas. Pour l'ensemble de la population c'est une perte égale à i 8 p. Ioo de l'effectif total théorique des femelles.

Dans les autres races françaises (Alpine chamoisée et Poitevine) on trouve également đes intersexués et des mâles stériles, ce qui laisse à penser qu'il s'agit du même facteur qui aurait migré. Comme la sélection en faveur des animaux mottes est différente suivant la race et suivant le sexe, les effets du gène d'intersexualité sont variables et pas toujours spectaculaires du fait de l'effectif réduit des troupeaux et du nombre important des produits réformés 2 à 4 semaines après la naissance (presque tous les mâles et deux tiers environ des femelles). Nous aborderons dans un autre article l'étude de l'équilibre de la fréquence génique du caractère "sans cornes " en fonction de la sélection pratiquée. De toute façon, il semble absurde de continuer une politique d'élevage qui favorise les animaux " nottes". Cette politique entraîne des réformes supplémentaires, limite les accouplements et, finalement, masque l'objectif essentiel de la sélection, à savoir l'amélioration génétique des performances laitières.

$$
\text { Recu pone publication en norembre } 1967 \text {. }
$$

\section{SUMMARY}

A SINGLE GENETIC HYPOTHESIS TO ACCOUNT FOR THE PRESENCE OF INTERSEXUAL ANIMALS, OF MALES IN EXCESS AND INFERTILE MALES IN "SAANEN " GOATS

In alpine breeds of goats, the polled character is linked with a form of hermaphrodism. In the previous literature, one autosomal gene was said to be responsible for the two phenomena: for polledness, it would have a dominant action with complete penetrance in both sexes ; for hermaphrodism, a recessive action limited to female goats. This explanation does not account for two others 
phenomena very often noticed in herds mated with polled sires, i. e. abnormal sex ratio, the female class being deficient, and male sterility. Soller et al. (I963) thought that infertile male goats are sometimes PP homozygotes. To account for the abnormal sex ratio, the Israeli authors (SoLLER and ANGEL, I964) were led to 3 hypotheses : that there was a direct recessive action of the intersexual gene on the sex ratio; that there was a greater fetal lethality of polled females; and lastly, that some homozygous PP females became thorough male animals.

We are inclined to the view that the only latter hypothesis can account for the presence of intersexual animals, the greater number of males, and the presence of infertile male goats, with the only supposition that there is a complete penetrance of the $\mathrm{P}$ gene in homozygous genetical females.

This hypothesis was statistically confirmed (see table 3 ) with the evidence that in matings of

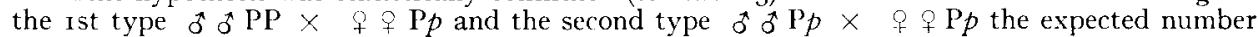
of females was reached - viz 24.0 and 39.9 per cent versus 25.0 and 37.5 per cent for the ist and 2 nd types respectively. There was a deficiency of intersexual animals -8.9 and 4.3 per cent versus 25.0 and $\mathrm{I} 2.5$ per cent - and an increase in males - 67.I and 55.8 versus 50.0 per cent. Moreover, an investigation on the male offspring of a homozygous PP sire showed that the number of sterile males does equal the number of males in excess, 26 . I versus 21.9 per cent (see table 4 ). To check completely the hypothesis, it is still necessary to demonstrate that both sterile males and intersexual animals have definite female $x x$ caryotypes.

Therefore, our findings confirm that polledness is induced by a dominant simple gene (table I) and that the heterozygous $P p$ females had a slight advantage on $p p$ females as regards fertility (table 2). We also find differences in fertility between males (PP versus $p p$, table 2 ) though not significant. More precise investigations on male fertility are required, in field service and above all in artificial insemination.

\section{RÉFÉRENCES BIBLIOGRAPHIQUES}

AsDelL S. A., 1944. The genetic sex of intersexual goats and a probable linkage with the gene for hornlessness. Science, N. Y., 99, 124.

ASDEll S. A., r946. Goat research in 1944-45. Yb. Br. Goat Soc., 29-30.

Asdell S. A., Bucinanan-Smith A. D., i 928 . Inheritance of color, beard, tassel and horns in the goat. J. Hered., 19, 425-430.

ISDELL S. A., Crew F. A. E., 1925. The inheritance of hom in the goat. J. Genet., 15, 367-374.

Basrur P. K., Covbrougit R. I., i964. Anatomical and cytological sex of a Saanen goat. Cytogenetics, 3, 4 I $4-427$

Buecin II. F., I957. Untersuchungen über das verschobene Geschlechtsverhältnis die Intersexualitët und die Fruchtbarkeit bei der Milchziege. Z. Tiersiicht. ZïchiBiol., 69, 30-90.

Cortefi, J. M., I066. Reproduction et insémination artificielle dans l'espèce caprine. Bull. tech. In/. Ingrs Servs Agric., (210), 463-47I.

liaton O. N., i94.3. An anatomical sturly of hermaphrodism in goats. Amer. J. vet. Res., 4, 333-343.

EATON O. N., I945. The relation between polled and hemaphroditic characters in dairy goat. Genetics, Princeton, 30, 5 I-6I.

Iiaton O. N., Simmons V. L., i939. Itemaphrodism in milk goats. J. Hered. 30, 26r-266.

If Unkouist H., 19,32. ['ber Vererbung von IIypospadie bei Ziegen. Biologia Gener., 8, 59-64.

Grouchy J. de, laiterone J.-J., Ricordeau (i., 1965. Études cytogénétiques chez i6 chèvres intersexuées, C. r. hebrl. Séanc, Icad. Sci., Paris, 260, 2932-2935.

ILAugen E., igho. Hermaphrodisme chez la chevre. Meld. Norg. LandbrHö̈sk, 39 ( Io), I-33 (en norvégien).

Konvo K., r949. (ienetic studies on the intersex in milk goat. I. An anatomical study of intersexual goats. Jap. J. Zootech. Sici, 19, 1-4.

Lior M., Barnea R., Angel H., Solder M., ig6z. Polledness and hemajhroditism in Saanen goats. Israel J. Agric. Re's, 12, $83-88$.

IöHLE K., BARFiss F., г 96 г. Untersuchungen über Körpergewichte und Körpermasse bei fruchtbaren und unfruchtbaren Ziegenbörken. Arch. Geflugel. Kleinlierk., 10, 12 1-1 28.

L.USH J. L., I926. Inheritance of horns, wattles, and color in grade Toggenburg goats. J. 11ered., 17, 7.3-91.

Nes N., Andersen K., Slagsvold I', 1963. Lixamen chromosomique de chèvres hermaphrodites. Hedlemsbl. Norske Veterinaerfor., 7, i $55^{-166}$ (en norvégien).

l'ADEII B., WYsoki M., Ayalox N., Soller M., ig65. An $x x / x y$ hermaphrodite in the groat. 1 srad $J$. med. Sci., 1, rooß-лог 2 .

Petitjean M.J., Cochez I.. P., ig66. A propos de la subfertilité de coqs homozygotes pour le gène " R " (crête rosacée). 13ih Wld's Poult. Congr., ı25-1 30. 
RICORDEAU G., I968. (En préparation).

Schönherr S., I956. Die Unfruchtbarkeit der Ziegenböcke, ihre Verbreitung, frühzeitige Erkennung und Bekämpfung. Z. Tierzüchl. ZüchtBiol., 66, 209-234, 381-415.

Solter M., Angel. II., I964. Polledness and abnormal sex ratio in Saanen goats. J. Hered. 55, 139-142

Soller M., Kempenich O., I964. Polledness and litter size in Saanen goats. J. Hered. 55, 301-304.

Soller M., Laor M., Barnea R., Weiss Y., Ayalon N., ig63. Polledness and infertility in male Saanen goats. J. Hered., 54, 237-240. 